\title{
Real time and online dynamic speckle assessment of growing bacteria using the method of motion history image
}

\author{
Mohammad Zaheer Ansari \\ Evelio E. Ramírez-Miquet \\ Isabel Otero \\ Dania Rodríguez \\ Juan G. Darias
}




\title{
Real time and online dynamic speckle assessment of growing bacteria using the method of motion history image
}

\author{
Mohammad Zaheer Ansari, ${ }^{a, *, \dagger}$ Evelio E. Ramírez-Miquet, ${ }^{b, *, \dagger}$ Isabel Otero, ${ }^{\mathrm{b}}$ Dania Rodríguez, ${ }^{\mathrm{b}}$ and \\ Juan G. Darias ${ }^{b}$ \\ aIndian School of Mines, Department of Applied Physics, Biomedical Optics Laboratory, Dhanbad, Jharkhand 826004, India \\ ${ }^{b}$ Centro de Aplicaciones Tecnológicas y Desarrollo Nuclear, Calle 30 No. 502, Miramar, C. P. 11300 La Habana, Cuba
}

\begin{abstract}
This paper reports the application of the motion history image (MHI) method for biospeckle processing of a bacterial growth. The method avoids the complexity as well as the large computation in sequence-matchingbased methods and detects whether the speckle structure has changed or not. Encouraging experimental results on the real-time evolution of the growing bacteria during $12 \mathrm{~h}$ demonstrate the effectiveness of the proposed method. The MHI presented an online result without loss of resolution and definition. In turn, the MHI also presented the ability to provide a close answer to the traditional offline method of generalized differences. $\odot 2016$ Society of Photo-Optical Instrumentation Engineers (SPIE) [DOI: 10.1117/1.JBO.21.6.066006]
\end{abstract}

Keywords: biospeckle; bacteria; motion history image; generalized differences.

Paper 160098R received Feb. 19, 2016; accepted for publication May 17, 2016; published online Jun. 8, 2016.

\section{Introduction}

Dynamic laser speckle ${ }^{1}$ is a promising optical technique for the evaluation of biological processes. Dynamic speckles arise after illuminating a sample undergoing an intrinsic activity with coherent light. The evolution of this activity is characterized by multiple descriptors, which provide qualitative and quantitative information on the processes causing dynamic changes in the sample under study.

Biospeckle implementation for following the evolution of growing bacteria is of actual relevance in various procedures in biomedical engineering, microbiology, and biotechnology. This potential implementation leads to processing optimization and reduced computation to conceive real-time assessment of microbial growth. Some methodologies employed in processing dynamic speckle stacks usually implicate that long monitoring time is needed before any assessment can be concluded and also require long processing time before obtaining an outcome from the calculations. Therefore, reducing the time required to process speckled images containing information on chemotaxis, microbial growth, pathogen inactivation, or their susceptibility (vulnerability) to specific antibiograms would contribute to rapid in vitro diagnosis and early identification of kinematic features of a particular pathogen. Moreover, biospeckle systems allow for a simple optical configuration capable of interrogating biosystems while maintaining the noncontact regime. ${ }^{2}$

Dynamic speckles have been previously employed to follow the chemotaxis of Pseudomonas aeruginos ${ }^{3}$, for temporal characterization of Escherichia coli (E. coli) in solid medium, ${ }^{4}$ and to easily discriminate between different microorganisms in the same culture. ${ }^{5}$ The activity of speckle patterns due to bacteria is

\footnotetext{
*Address all correspondence to: Mohammad Zaheer Ansari, E-mail: mohamedzaheer1@gmail.com; Evelio E. Ramírez-Miquet, E-mail: ermiquet@ceaden.edu.cu

†These authors contributed equally to this paper.
}

manifested as motility, multiplication, and spreading of colonies. ${ }^{6,7}$ Recently, the effects of continuous and instantaneous laser exposure and intensity on the spreading rate of bacterial colonies and yeast were explored. ${ }^{8,9}$ Bacterial spreading rate is a key parameter for quality control of possible contaminated samples. ${ }^{10}$ An optimal image processing allowing a rapid diagnosis is crucial in online assessment of bacterial colonization in biofilms, water, or food. The optimization of biospeckle processing methods provides an alternative tool for real-time implementation of dynamic laser speckle techniques in microbiology, biotechnological work, and customs inspection routines.

This paper proposes the motion history image (MHI) method applied to speckle pattern sequences to follow the bacterial spreading in real time. We analyze its suitability as compared to another established method for biospeckle image processing: the generalized differences (GD). A temporal characterization of the bacterial spreading is presented using the absolute value of the differences (AVD). The analysis presented here may provide a tool for online assessment of growing microorganisms, in particular, for the evaluation of bacterial growth.

\section{Methods}

\subsection{Theory}

MHI is a method used to identify movements within a row of images in time. This technique creates the final image by following the rule that each pixel $(x, y)$ in the MHI is marked with the timestamp (hour, minute, second, and millisecond) and will be removed if it does not vary after a certain time parameter, called the lifetime or duration of MHI. ${ }^{11,12}$

The MHI represents a static image template, where the pixel intensity is a function of the recency of motion in an image sequence. This single image appears to contain the necessary 
information for determining how an object has moved during the action. In our approach, we collapse the temporal motion information into a single image template, where intensity is a function of the recency of motion.

Figure 1(a) shows an image of a hand, where its movement will help us understand the construction of MHI related to changes in the image throughout space [see Fig. 1(b)]. When there is a movement of the hand as shown in Fig. 1(b), the collection of images can be manipulated by the MHI procedure in several steps, as follows. Successive images of the object showing movements are compared using the following equation: ${ }^{13,14}$

$S_{j}=I_{j}-I_{j-1}$,

where $I_{j-1}$ and $I_{j}$ are the grayscale images in the moments $j-1$ and $j$. In the resultant image, called the silhouette image, the threshold function ${ }^{13,14}$ presented by Eq. (2) is applied:

$T_{j}(x, y)=\left\{\begin{array}{ll}1, & \text { if } S_{j}(x, y)>Z \\ 0, & \text { if } S_{j}(x, y) \leq Z\end{array}\right.$,

where $Z$ is the threshold parameter and $T_{j}(x, y)$ is the threshold image at each moment $j$.

Finally, the MHI procedure is the weighting of the threshold images within the buffer of size $n$ by a constant representing the lifetime in the moment $j$, as given in Eq. (3):

$\operatorname{MHI}(x, y)=\sum_{j=1}^{n} T_{j} k_{j}$.

The value of the constant $k_{j}$ will be

$k_{j}=\left\{\begin{array}{cc}0, & \text { imagelifetime }>\text { MHI duration } \\ \frac{\text { imagelifetime.255 }}{k_{n}}, & \text { otherwise }\end{array}\right.$,

where the variable imagelifetime represents the lifetime of the image in the moment $j$.

In Fig. 1(c), it is possible to see the result of the motion carried by the hand in a static representation of MHI. We can observe the trail of the movement made by the hand, where the final motion locations appear red in the MHI static image. The images presented to explain the method of MHI were captured with authorization of the institutions where authors are affiliated.

The MHI results were compared with routine offline methods of $\mathrm{GD}^{15}$ and Fujii, ${ }^{14}$ as presented in Eq. (5) and (6):

$\mathrm{GD}(i, j)=\sum_{k} \sum_{l}\left|I_{k}(i, j)-I_{k+l}(i, j)\right|$,

where $I_{k}(i, j)$ is the intensity matrix with $(i, j)$ representing the coordinates in the $k^{\prime}$ th frame and the bars indicating absolute value:

$F(i, j)=\sum_{k} \frac{\left|I_{k}(i, j)-I_{k+1}(i, j)\right|}{\left|I_{k}(i, j)+I_{k+1}(i, j)\right|}$.

\subsection{Numerical Evolution of Absolute Value of Differences}

Images of biological material under laser illumination can be analyzed numerically via the creation of the time history speckle pattern (THSP). ${ }^{16}$ By choosing repeatedly a particular row or column in the speckle images at a certain sampling rate and then placing these strips side by side, one can make a standard THSP image. Based on the dynamic speckle behavior of an object under coherent illumination and with the corresponding THSP image formed, it becomes possible to estimate the degree of activity of the specimen. One of the calculations used to measure the activity of the biological media from THSP images is the AVD. ${ }^{17}$ The AVD processing is based on the generation of a single numeric value from THSP.

For the numerical analysis, active zones of the sample taken as the region of interest were analyzed with respect to their behavior in time by means of the AVD method. ${ }^{17,18}$ The AVD computation can be expressed by

$\mathrm{AVD}=\sum_{i j} \mathrm{COM}_{i j}|i-j|$

where $\mathrm{COM}$ is the co-occurrence matrix related to the THSP, ${ }^{19-21}$ and $i$ and $j$ variables define the dimension of the COM matrix.
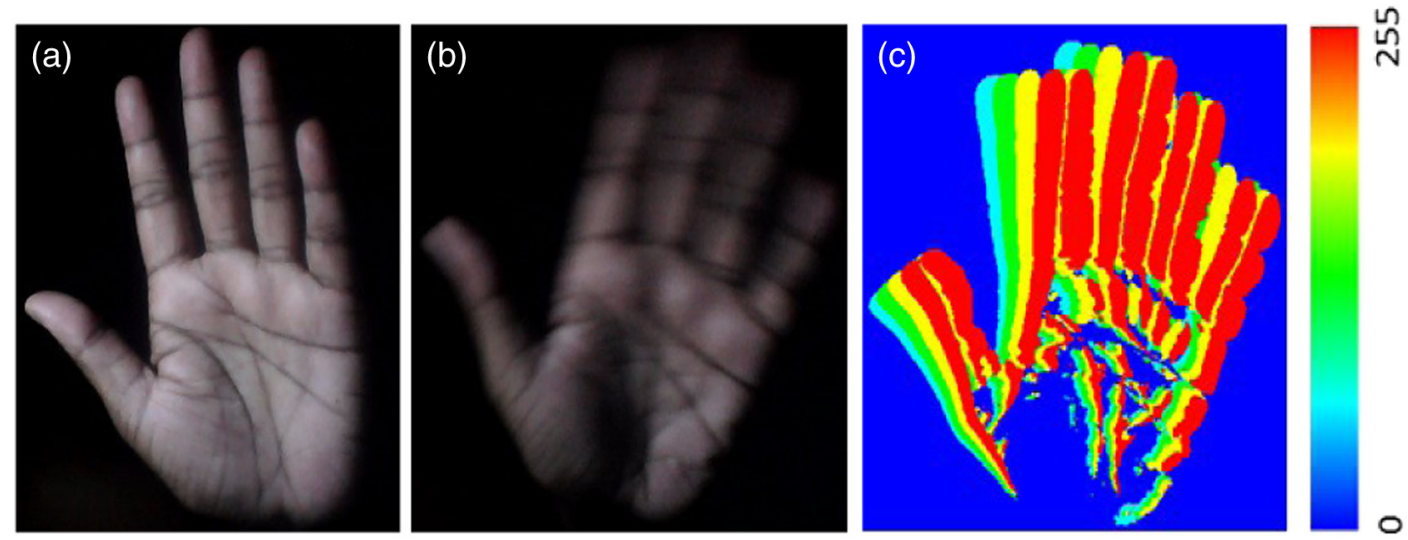

Fig. 1 (a) Image of the hand, (b) movement in the hand, and (c) motion history. The image was formed by taking the threshold of the subtraction of images stored in the buffer of the hand in a downward movement, where the final motion locations appear in red in the MHIs. 


\section{Experiments}

The global idea of our experiments consists in following growing Staphylococcus aureus (S. aureus) in time during $12 \mathrm{~h}$. We performed a series of twenty assays aimed at reducing the exposure time of bacteria as much as possible. Another 20 replications were performed in which exposure of bacteria was continuous during the monitoring time.

\subsection{Preparation of the Assay}

The culture medium (Tryptone Soy Agar) was prepared according to standard procedures established in routine protocols of manufacturers. Ten milliliters of warm agar was poured inside Petri plates and cooled for $\sim 10 \mathrm{~min}$. As the culture medium solidifies, the concentration of bacteria is determined using a spectrophotometer (T60U from PG Instruments Limited) allowing turbidimetric measurement of bacterial density. The values measured in the spectrophotometer were previously correlated to bacterial concentration via a viability assay performed during a calibration process based upon a standard plate count in solid agar. Every assay was carried out using an initial concentration of $S$. aureus ATCC 13565 equal to $10^{6} \mathrm{CFU} / \mu \mathrm{L}$. A single drop of $5 \mu \mathrm{L}$ of bacteria in the logarithmic phase was inoculated in the solid culture and placed inside an incubator (Memmert INE 400) with fixed temperature at $37 \pm 0.5^{\circ} \mathrm{C}$. Following the protocols of biosecurity and biocontrol, the seeding procedures were completed inside a laminar flow hood.

\subsection{Experimental Setup}

The system is described in Fig. 2. The incubator (1) contains the optical setup, and the sample (2) is located over a sample holder aligned with the visualization system. A laser diode (3) (Hitachi HL6738MG, emitting at $7 \mathrm{~mW}$, 680-nm wavelength) is employed as a coherent wave source. A shutter (4) is used in half of the experiments to interrupt the laser beam, so that the sample is exposed only when a speckled frame is to be recorded. The photographic objective (5) (Industar $61 \mathrm{~L} / \mathrm{D}$, focal distance $18 \mathrm{~mm})$ creates a sharp image $(3 \mathrm{~cm} \times 2 \mathrm{~cm})$ of the illuminated surface on the CCD array of an NGC Technology Swift Cam 300 USB 2.0 camera (6). Images from the camera are registered in a computer (7) via a USB interface.

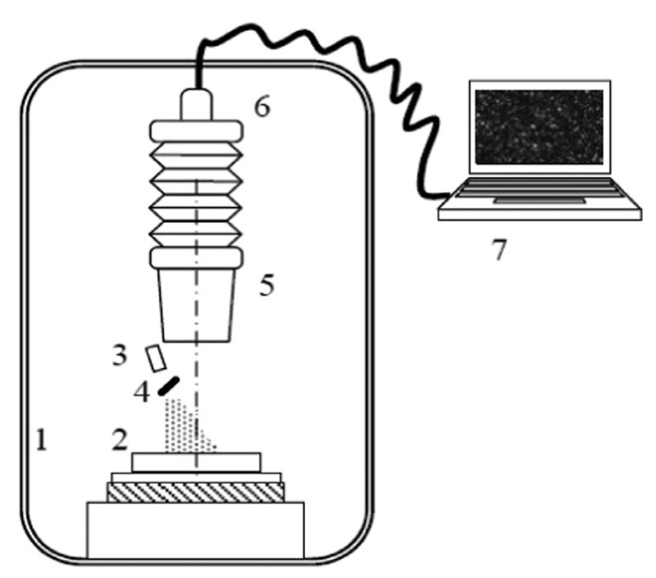

Fig. 2 Setup for experimental assays with $S$. aureus: (1) incubator, (2) sample, (3) laser diode, (4) shutter, (5) photographic objective, (6) camera, and (7) computer.

\section{Results and Discussion}

When growing bacteria, $S$. aureus is illuminated with a laser, a speckle pattern is obtained, and a sequence of images is registered. After processing the dynamic speckle signals using the method of MHI, it becomes easier to identify different degrees of motility in real time during its growth. The application of MHI in a process of biological activity of growing bacteria, S. aureus, during $2 \mathrm{~h}$ of evolution is shown in Fig. 3(a). As can be seen from Fig. 3(a), the MHI image preserves both the resolution and dimension of the image. Moreover, the applicability of the MHI procedure was conducted by comparison with a well-known offline method. In Fig. 3(b), it is possible to see the GD method, where the activity of the bacteria is shown. However, in the Fujii method, not shown here, the activity regions were not so identified due to its ability to highlight the activities in the darker zones.

Figure 4 shows the areas of activity in pseudocolor images that result from the MHI method and the GD method. During $4 \mathrm{~h}$ of the evolution of the $S$. aureus, there was an effect that could be detected by following the activity maps. The intense change in the activity presented a higher density of dots, while low activity was lighted with a smaller density of dots in the final processed MHI image [Fig. 4(a)]. The background was presented in blue as expected. Moreover, as can be seen from Fig. 4(b), the MHI followed the same patterns of the GD method.

Figure 5 illustrates the evolution of bacterial growth during $8 \mathrm{~h}$ of inoculation processed with MHI and GD methods. Again, the MHI presented its close behavior to GD processing without loss of resolution and dimension.

Figure 6 presents the pseudocolor images showing evolution of $S$. aureus growth during $12 \mathrm{~h}$ of inoculation processed using the MHI and GD algorithms. MHI presented the activity image of resolution and dimension close to the GD method.

Therefore, on following the MHI activity images, it becomes possible to illustrate a temporal evolution of the growth of $S$. aureus bacteria in real time. A little activity in the bottom left of the images is associated with a wet zone of the agar.

However, as per definition, the GD method loses the time dimension and so avoids a direct measure in time. In order to obtain the time evolution of the changes in bacterial growth using the GD measure, one must have to split the stack of dynamic speckle images into several substacks of images and to generate the GD maps for each substack. However, this splitting into substacks would lead to several GD maps, which in turn avoids a simple and fast assessment of the changes during bacterial growth.

Our proposed method of MHI appears as being an online and real-time tool in the evolution of bacterial growth with a high resolution and dimension, which is of utmost use for biomedical or biotechnological works.

Further, due to these promising results, we have assessed the biospeckle activity of the growing $S$. aureus bacteria using the numerical processing of the AVD method. Figure 7 shows the temporal evolution of the activity on growing bacteria during $12 \mathrm{~h}$ of inoculation. As can be seen from Fig. 7, the activity quantified by AVD increases rapidly along time, reaching a maximum during $12 \mathrm{~h}$.

In our previous paper, ${ }^{8}$ we presented an analysis of the diameter of the active zone obtained with a GD processing of biospeckle images of E. coli. The analysis of the diameter of growth versus time correlated to the regime of illumination yielded a 

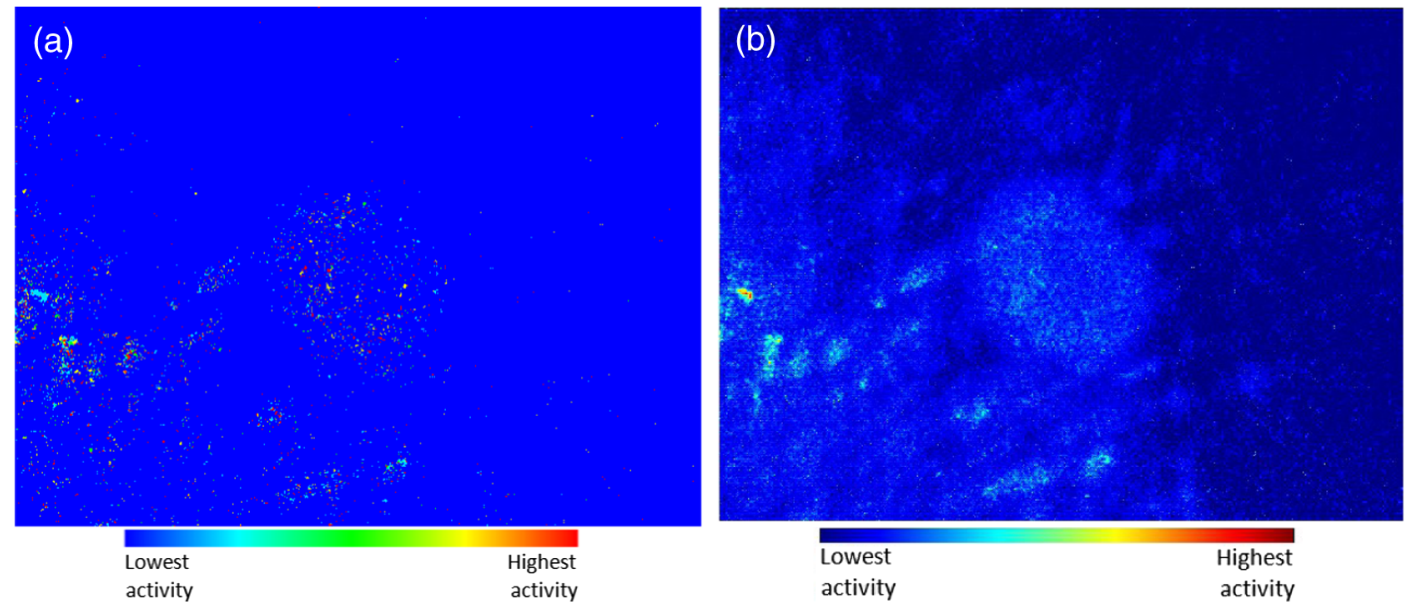

Fig. 3 Dynamic laser speckle of $S$. aureus during $2 \mathrm{~h}$ of evolution and processed with (a) the MHI and (b) GD methods.
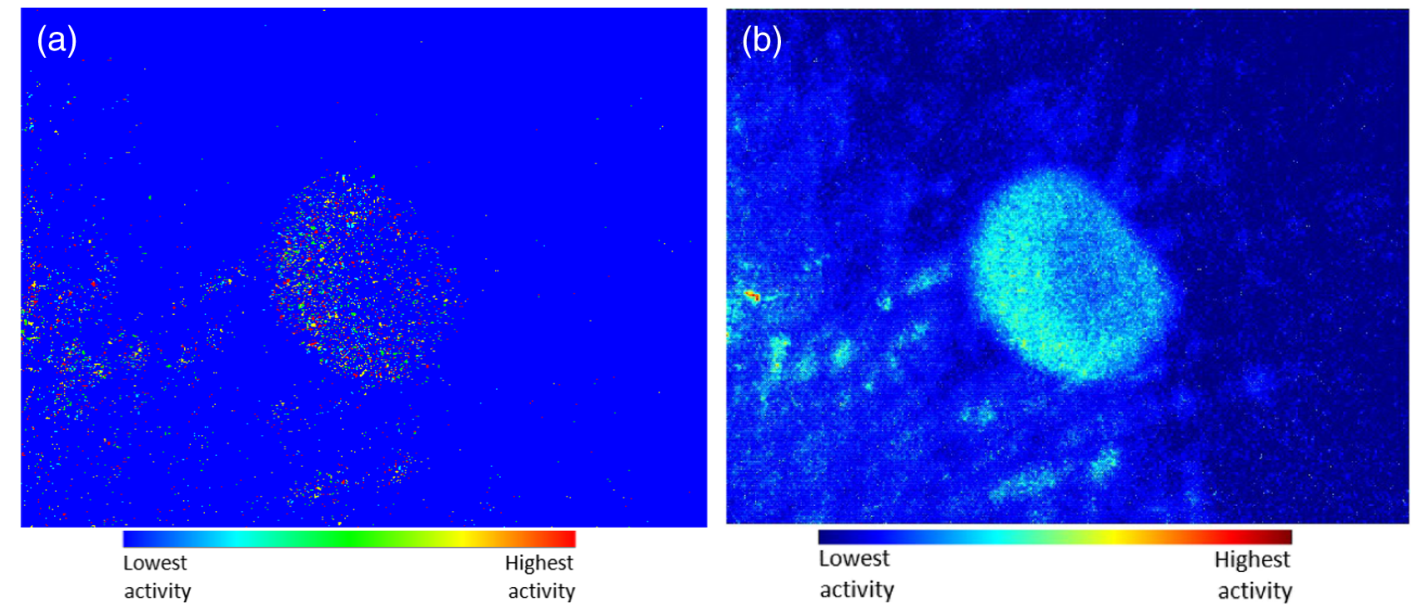

Fig. 4 Dynamic laser speckle of $S$. aureus during $4 \mathrm{~h}$ of evolution and processed with (a) the MHI and (b) GD methods.
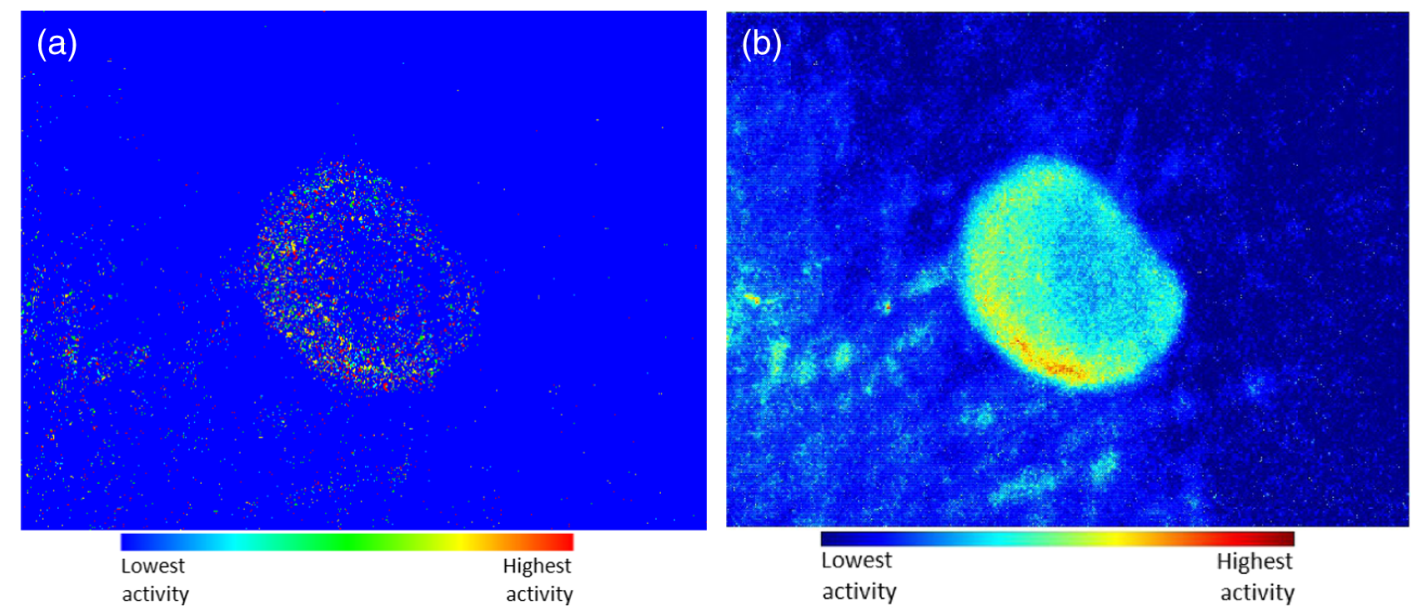

Fig. 5 Dynamic laser speckle of $S$. aureus during $8 \mathrm{~h}$ of evolution and processed with (a) the $\mathrm{MHI}$ and (b) GD methods. 

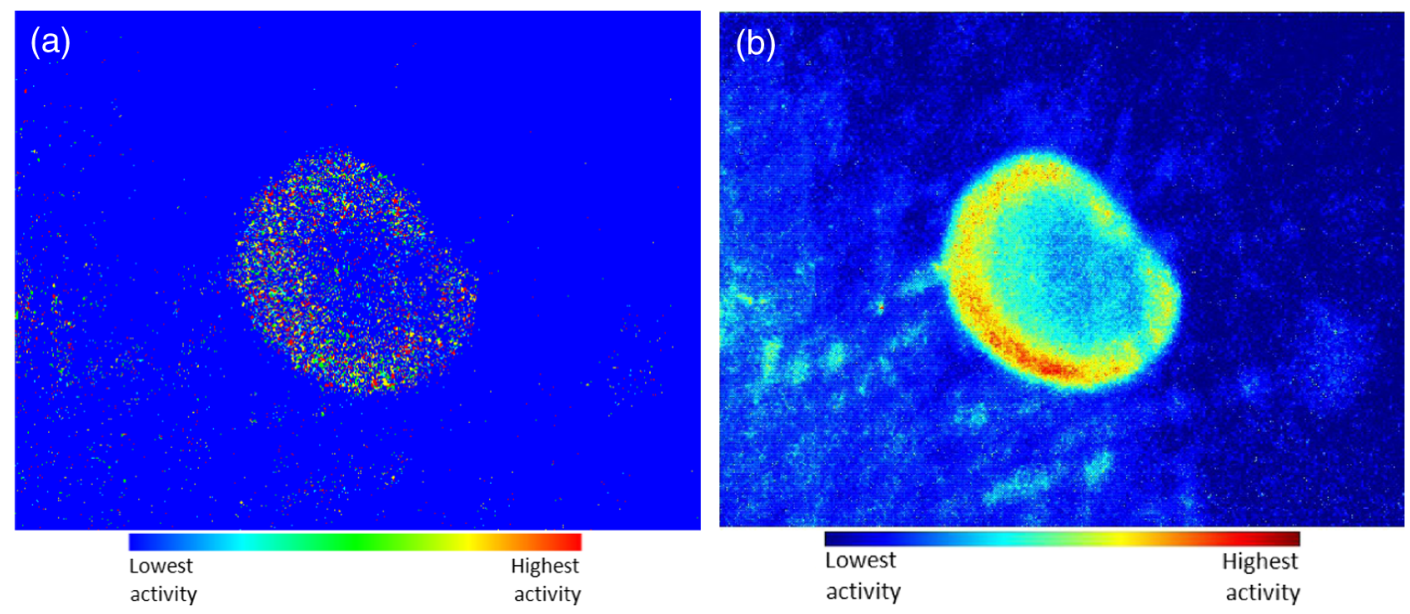

Fig. 6 Dynamic laser speckle of $S$. aureus during $12 \mathrm{~h}$ of evolution and processed with (a) the MHI and (b) GD methods.

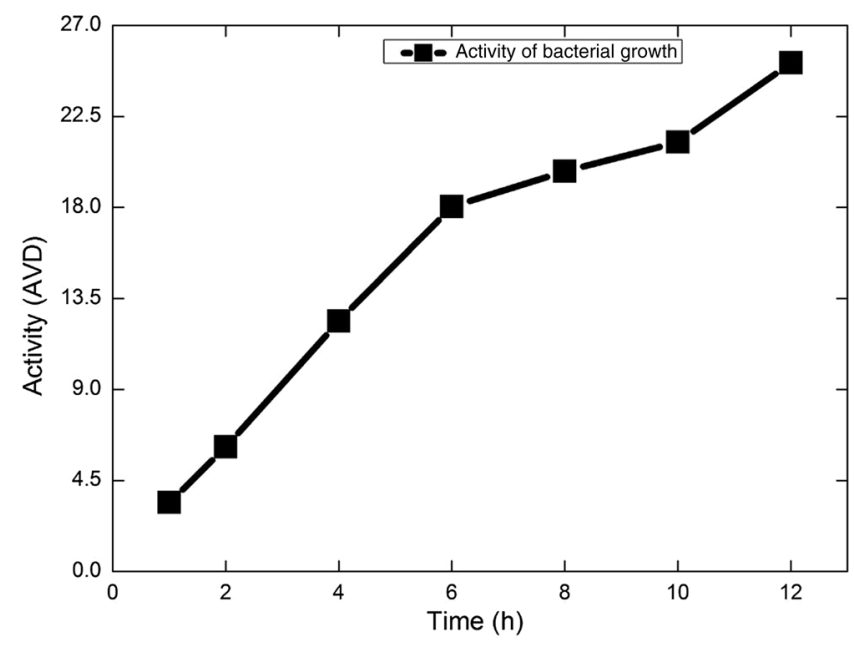

Fig. 7 Temporal evolution of the biological activity of growing bacteria, $S$. aureus, quantified by AVD over a time period of $12 \mathrm{~h}$. All values are normalized by $10^{-2}$.

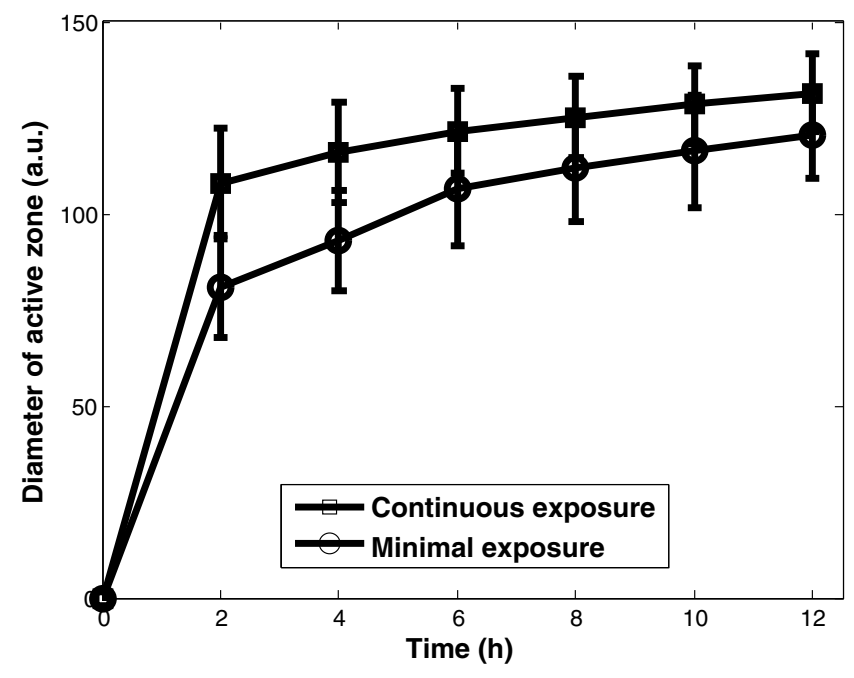

Fig. 8 Diameter of growth versus time for growing bacteria, S. aureus. graph with separated error bars that necessitated a statistical analysis to conclude further if continuous and instantaneous exposure affected the bacterial growth kinetics. Given the evident analogy of the results obtained after applying MHI and the GD methods, we proceeded to evaluate the influence of the illumination regime in the active zone in the biospeckle stacks using the outcomes from the MHI. Figure 8 shows the plot of the diameter of the active zone versus time for all experiments with $S$. aureus illuminated with continuous and instantaneous coherent light. The diameter of the region where bacteria spreads is quantified in terms of pixels in the horizontal axis, thus allowing the interpretation of possible effects of continuous laser exposure in the growth rate of bacteria. As can be appreciated in the graph, error bars overlap and, consequently, no significant impact on the spreading rate of $S$. aureus can be attributed to the laser, at least using this approach for the assessment of growth.

\section{Conclusions}

We have tested the MHI as an online and real-time algorithm for biospeckle monitoring of bacterial growth. A good correlation was achieved when MHI results were further compared to the generalized differences routine method for biospeckle processing. Our results suggest that the analysis presented here may provide a tool for online assessment of growing microorganisms, in particular, for the evaluation of bacterial growth.

\section{Acknowledgments}

The authors sincerely thank Professor Roberto A. Braga (Universidade Federal de Lavras, Brazil) for his fruitful help and discussions.

\section{References}

1. H. J. Rabal and R. A. Braga Jr., Dynamic Laser Speckle and Applications, CRC Press, Boca Raton (2009).

2. M. Z. Ansari et al., "Real time monitoring of drug action on T. cruzi parasites using a biospeckle laser method," Laser Phys. 26, 065603 (2016).

3. S. E. Murialdo et al., "Analysis of bacterial chemotactic response using dynamic laser speckle," J. Biomed. Opt. 14(6), 064015 (2009).

4. E. E. Ramírez-Miquet et al., "Escherichia coli activity characterization using a laser dynamic speckle technique," Rev. Cub. Fis. 28(1E), 1E13-1E17 (2011). 
5. S. E. Murialdo et al., "Discrimination of motile bacteria from filamentous fungi using dynamic speckle," J. Biomed. Opt. 17(5), 056011 (2012).

6. H. Sendra et al., "Dynamic laser speckle to detect motile bacterial response of Pseudomonas aeruginosa," J. Phys. Conf. Ser. 90, 012064 (2007).

7. C. Kaito and K. Sekimizu, "Colony spreading in Staphylococcus aureus,” J. Bacteriol. 189, 2553-2557 (2007).

8. E. E. Ramírez-Miquet et al., "Biospeckle technique for monitoring bacterial colony growth with minimal photo-exposure time associated," IFMBE Proc. 49, 313-316 (2015).

9. F. M. Vincitorio et al., "Dynamic speckle study of microbial growth," Proc. SPIE 9660, 96601Q (2015).

10. M. M. Lobete et al., "Recent trends in non-invasive in situ techniques to monitor bacterial colonies in solid (model) food," Front. Microbiol. 6, 148 (2015).

11. J. W. Davis, "Hierarchical motion history images for recognizing human motion," in Proc. of the IEEE Workshop on Detection and Recognition of Events in Video, pp. 39-46 (2001).

12. A. Bobick et al., "Real-time recognition of activity using temporal templates," in Proc. 3rd IEEE Workshop on Applications of Computer Vision, pp. 39-42 (1996).

13. R. C. Gonzalez et al., Digital Image Processing, Prentice Hall, New Jersey (2008).

14. R. P. Godinho et al., "Online biospeckle assessment without loss of definition and resolution by motion history image," Opt. Lasers Eng. 50(3), 366-372 (2012).

15. R. Arizaga et al., "Display of local activity using dynamic speckle patterns," Opt. Eng. 41(2), 287-294 (2002).

16. R. A. Braga et al., "Reliability of biospeckle image analysis," Opt. Lasers Eng. 45, 390-395 (2007).

17. R. A. Braga et al., "Evaluation of activity through dynamic laser speckle using the absolute value of the differences," Opt. Commun. 284, 646-650 (2011).

18. R. J. Gonzalez-Peña et al., "Monitoring of the action of drugs in melanoma cells by dynamic laser speckle," J. Biomed. Opt. 19(5), 057008 (2014).
19. A. Oulamara et al., "Biological activity measurement on botanical specimen surfaces using temporal decorrelation effect of laser speckle," J. Mod. Opt. 36(2), 165-179 (1989).

20. Z. Xu, "Temporal and spatial properties of time-varying speckles of botanical specimens," Opt. Eng. 34(5), 1487-1502 (1995).

21. R. Arizaga et al., "Speckle time evolution characterization by the co-occurrence matrix analysis," Opt. Laser Technol. 31(2), 163-169 (1999).

Mohammad Zaheer Ansari is a researcher at the Indian School of Mines, Dhanbad, Jharkhand, India. He received his bachelor's and master's degrees in physics from Vinoba Bhave University, Hazaribag (Jharkhand), India, in 2004 and 2007, respectively, and his MPhil and $\mathrm{PhD}$ degrees in applied optics from the Indian School of Mines, Dhanbad, India, in 2008 and 2016, respectively. He is the author of 15 scientific journal papers. His current research interests include biospeckle/dynamic speckles and their applications in agriculture and biology and biomedical optics.

Evelio E. Ramírez-Miquet is a researcher at the Centro de Aplicaciones Tecnológicas y Desarrollo Nuclear in Havana, Cuba. He received his bachelor's degree in nuclear engineering in 2010 and is currently pursuing his PhD in photonics and optoelectronic systems. His research interests include optical instrumentation and laser biospeckle for online assessment of dynamic processes related to biomedical engineering.

Juan G. Darias received his diploma in physics from the University of Havana in 1973. He worked as a lecturer and research assistant at the Higher Institute of Technologies and Applied Sciences (InSTEC) in Havana. He was the director of the Centro de Aplicaciones Tecnológicas y Desarrollo Nuclear (CEADEN) from 2003 to 2009. His current fields of interest are in nanotechnology, laser radiation detectors, laser speckle and mass spectrometry.

Biographies for the other authors are not available. 